\title{
Analysis of Neutrino Burst from the Supernova in the Large Magellanic Cloud
}

\author{
Hideyuki Suzuki and Katsuhiko Sato \\ Department of Physics, Faculty of Science, University of Tokyo \\ Bunkyo-ku, Tokyo 113, Japan
}

A massive star has been believed to end his life with the collapsed driven supernova explosion and the formation of the compact object such as a neutron star or a black hole. When the compact object is formed, a large amount of energy corresponding to the binding energy of the object must be released. It has been considered that most of the energy is emitted by neutrinos because of their adequate coupling with the matter. The observation of the neutrino burst from SN1987A by Kamiokande[1] and IMB[2] offered us the first chance to test these scenarios of the collapse driven supernova explosion directly. We began to analyze the data just after their publication and got many important results which are presented below. In our analysis the distance of SN1987A is assumed to be $50 \mathrm{kpc}$.

The First 2 Events : In all the detected 19 events( Kamiokande : 11, IMB : 8), only the first 2 events of the Kamiokande data may be scattering events, that is, $\nu_{\mathrm{e}}+\mathrm{e}^{-} \longrightarrow \nu_{\mathrm{e}}+\mathrm{e}^{-}$. One may consider that these 2 events due to the neutronization burst of $\nu_{e}$. Taking into account the small cross section of the scattering, however, it is doubtful to treat them as the scattering events. We estimate the energy of $\nu_{\mathrm{e}}$ corresponding to the first 2 events and got the large value, $1.9 \cdot 10^{53} \mathrm{erg}[3]$. In all simulations ever published, the energy corresponding to the neutronization burst is the order of $10^{51} \mathrm{erg}$ and the expected number of events in the Kamiokande detector is less than 0.04 . Furthermore the duration time of the neutronization burst is about $10 \mathrm{msec}$ while the second event occurred $100 \mathrm{msec}$ after the first one. Hereafter we treat the all events as ones due to the reaction $\bar{\nu}_{\mathrm{e}}+\mathrm{p} \longrightarrow \mathrm{e}^{+}+\mathrm{n}$.

Neutrino Temperature : Assuming the neutrino spectrum to be Fermi-Dirac distribution with the vanishing chemical potential, we got the $\bar{\nu}_{\mathrm{e}}$ temperature, $T_{\nu}[3,4]$. For the Kamiokande data, $T_{\nu}$ is $2.6 \sim 3.1 \mathrm{MeV}$, for IMB $3.9 \sim 5.3 \mathrm{MeV}$. These values are a little close to the values by those who insist the late time neutrino heating mechanism of the explosion.

Total Energy of Neutrinos and Mass of Neutron Star : Using above neutrino temperature, we can estimate the total energy emitted by neutrinos as six times $\bar{\nu}_{\mathrm{e}}$ energy[4]. It becomes very reasonable value, $2.9_{-0.4}^{+0.6} \cdot 10^{53} \mathrm{erg}$ for the Kamiokande data and $1.5_{-0.6}^{+1.2} \cdot 10^{53} \mathrm{erg}$ for IMB. This energy can be interpreted as the binding energy of the remnant neutron star. Once we know 
the binding energy of the neutron star, we can determine the mass of the neutron star. Due to the ambiguity of the equation of state and the small statistics of the data, we cannot specify one value for the mass. But we may conclude that a neutron star whose mass is in the range of $0.6 \sim 1.9 M_{\odot}$ has been formed by SN1987A not a black hole (see Figure 1).

Bunched Structure of the Data : Kamiokande and IMB data show some bunched structure. We calculate the neutrino temperature and the the radius of the neutrinosphere for each bunch[5]. The first 8 events of Kamiokande and the first 6 events of IMB can be well understood in the scenario of the protoneutron star contraction. That is, the protoneutron star born with the radius of several times $10 \mathrm{~km}$ contracts to the normal neutron star whose radius is about $10 \mathrm{~km}$ in a few seconds and in this stage neutrino temperature once increases with the reduction of the volume.

The Last 3 Events : As described above, whole feature of the neutrino burst is well explained by the standard model. But the last 3 events of Kamiokande which occurred after the $7 \mathrm{sec}$ gap is mysterious. For example, the corresponding energy is large and the radius of the neutrinosphere of several times $10 \mathrm{~km}$ is necessary for this energy[5].

The concept that neutrinos diffuse out of the supernova core carrying out its thermal energy is confirmed by the observation of the neutrino burst from SN1987A for the first time. But we must keep in mind that there are some puzzles such as the last 3 events of Kamiokande. There may exist some unknown mechanism of neutrino emission at the late time.

\section{References}

[1] K. Hirata et al., Phys. Rev. Lett. 58 (1987) 1490.

[2] R. Bionta et al., Phys. Rev. Lett. 58 (1987) 1494.

[3] K. Sato and H. Suzuki, Phys. Rev. Lett. 58 (1987) 2722.

[4] K. Sato and H. Suzuki, Phys. Lett. in press.

[5] H. Suzuki and K. Sato, Publ. Astron. Soc. Japan 39 (1987) 521.

\section{Figure 1:}

The mass of neutron star vs. the binding energy for several EOS.

$\mathrm{M}_{\mathrm{G}}$ in units of $M_{\odot}$ $E_{b}$ in units of $10^{53} \mathrm{erg}$

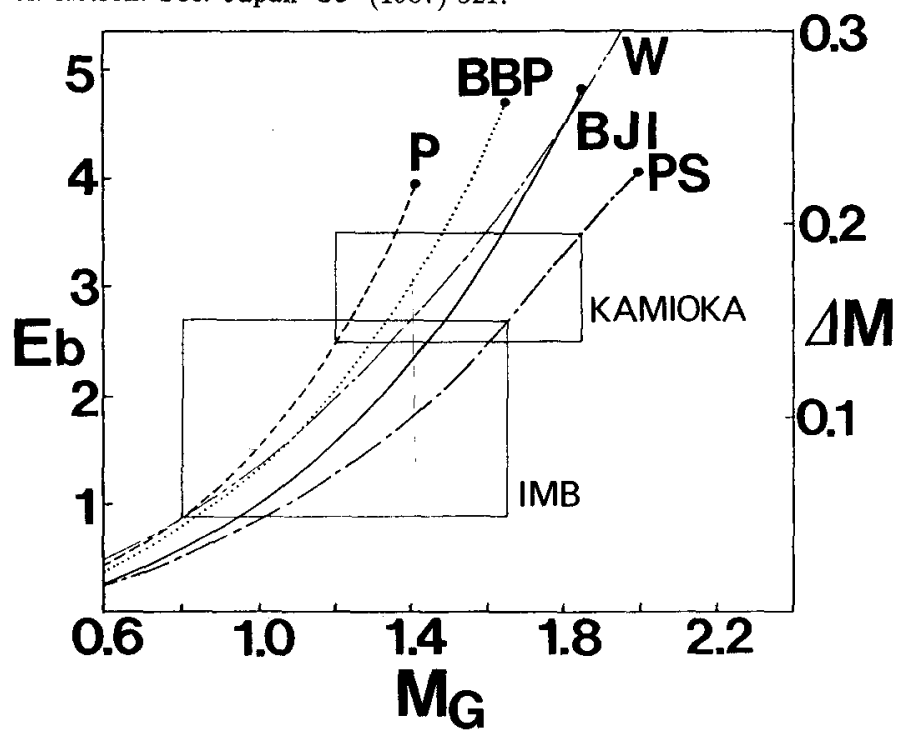

